\title{
Impact of thoracic epidural sympathetic block on cardiac repolarization
}

This article was published in the following Dove Press journal: Local and Regional Anesthesia

\author{
Makoto Komatsuzaki \\ Toshifumi Takasusuki \\ Shigeki Yamaguchi \\ Department of Anesthesiology, \\ Dokkyo Medical University, School \\ of Medicine, Mibu, Tochigi 321-0293, \\ Japan
}

Purpose: The interval from the peak to the end of the $\mathrm{T}$ wave ( $\mathrm{Tp}-\mathrm{Te}$ ) on electrocardiography is considered a marker of ventricular arrhythmias. A previous study suggested that right stellate ganglion block prolonged QT and QT dispersion (QTD). We investigated the effect of thoracic epidural sympathetic block with 1\% mepivacaine on QT, QTD, Tp-Te, and Tp-Te/QT by using computerized measurement.

Patients and methods: After obtaining the approval of the ethics committee of Dokkyo Medical University Hospital, 23 patients with American Society of Anesthesiologists physical status I or II who were scheduled to undergo thoracic surgery were enrolled. An epidural catheter was inserted at the Th4 5 or 5-6 level and then used for injection of $7 \mathrm{~mL}$ of $1 \%$ mepivacaine. Changes in RR interval, QT, corrected QT (QTc), QTD, QTc dispersion (QTcD), Tp-Te, Tp-Te/ $\mathrm{QT}$, and Tp-Te/QTc before and after epidural injection were assessed by computerized measurement. Statistical analysis was performed by one-way ANOVA.

Results: Systolic blood pressure was consistently suppressed 10-15 minutes after injection (baseline: $136 \pm 10 \mathrm{mmHg}, 11$ minutes: $113 \pm 12 \mathrm{mmHg}, 12$ minutes: $112 \pm 13 \mathrm{mmHg}, 13$ minutes: $112 \pm 12 \mathrm{mmHg}, 14$ minutes: $108 \pm 17 \mathrm{mmHg}, 15$ minutes: $111 \pm 14 \mathrm{mmHg} ; P<0.05)$. However, RR interval, QT, QTc, QTD, QTcD, Tp-Te, Tp-Te/QT, and Tp-Te/QTc were not changed after epidural block.

Conclusion: Thoracic epidural injection of 1\% mepivacaine did not alter QT, QTc, QTD, QTcD, Tp-Te, Tp-Te/QT, or Tp-Te/QTc. These results emphasize the safety of thoracic epidural sympathetic block with $1 \%$ mepivacaine for patients compared with right stellate ganglion block, in terms of cardiac repolarization.

Keywords: thoracic epidural block, QT interval, QT dispersion, Tpeak-Tend, mepivacaine

\section{Introduction}

Thoracic epidural block is widely used for chronic pain syndromes such as neuropathic pain or postoperative pain after thoracotomy. A previous work revealed that right stellate ganglion block (SGB), which produces a cervicothoracic sympathetic block, significantly prolonged QT and QT dispersion (QTD), compared with left SGB. ${ }^{1}$ In contrast, left SGB shortened QT and QTc interval; thus, it is used for the treatment of idiopathic long QT syndrome. Notably, QTD, defined as the difference between the maximal and minimal QT interval on a 12-lead surface electrocardiogram (ECG), reflects the regional heterogeneity of ventricular repolarization and has been established as a reliable parameter to predict cardiovascular events. Thus, right SGB affects ventricular repolarization, which may lead to life-threatening arrhythmias. Although the effects of SGB on QT and QTD are well established, the effects of thoracic epidural
Correspondence: Toshifumi Takasusuk Department of Anesthesiology, Dokkyo Medical University, School of Medicine, Kitakobayashi 880, Mibu, Tochigi 3210293, Japan

Tel $+8 \mid 282860788$

Fax +8I 282860478

Email takasusu@dokkyomed.ac.jp 
sympathetic nerve block on cardiac repolarization remain uncertain.

Prolongation of the interval between the peak and end of the $\mathrm{T}$ wave ( $\mathrm{T}$ peak-T end: $\mathrm{Tp}-\mathrm{Te}$ ), which may reflect the transmural dispersion of ventricular repolarization (TDR), has also been associated with ventricular arrhythmias. ${ }^{2-4}$ The Tp-Te/QT ratio is also known as a crucial marker of TDR. ${ }^{4}$ A previous study reported that epidural anesthesia, induced by $0.5 \%$ bupivacaine (an anesthetic concentration), reduced the QT interval and Tp-Te (a marker of TDR). ${ }^{5}$ Although the effect on QT dispersion and Tp-Te during thoracic epidural block by an anesthetic dose of bupivacaine has been established, the effect of thoracic block by local anesthetics, which block the sympathetic nerve, has not been elucidated.

To determine the influence of thoracic epidural sympathetic block on cardiac repolarization, we prospectively evaluated the QTD, Tp-Te, and Tp-Te/QT before and after epidural injection of $1 \%$ mepivacaine.

\section{Patients and methods}

Twenty-three patients, aged 20-60 years, with American Society of Anesthesiologists physical status I or II who were scheduled to undergo thoracic surgery were included. This study was approved by the ethics committee of Dokkyo Medical University, and all patients provided written informed consent. The study was registered at the UMIN Clinical Trials Registry (registration number: UMIN000029180). Patients with cardiovascular, respiratory, metabolic, and cerebrovascular diseases, as well as those with ECG abnormalities, were excluded from this study. No patient received antiarrhythmic drugs with a possible risk of QT prolongation. No premedication was given to any of the patients.

One day prior to the operation, each patient underwent installation of a thoracic epidural catheter. Standard 12-lead ECGs (FDX-4521L; Fukuda Denshi Co. Ltd, Tokyo, Japan) and noninvasive arterial blood pressure were monitored. Patients were placed in a sitting position on the table and they underwent insertion of catheter with a Tuohy needle at Th4/5 or 5/6. The catheter was advanced $5 \mathrm{~cm}$ in the cephalad direction. After an aspiration test for blood and cerebrospinal fluid, a test dose with $3 \mathrm{~mL}$ of $1 \%$ mepivacaine was injected through the catheter. The patient was observed in the supine position for 10 minutes after the test dose; subsequently, 7 $\mathrm{mL}$ of $1 \%$ mepivacaine was injected through the catheter. The anesthetic area was assessed using cold sensation to determine which dermatome levels were covered. Simultaneously, the laterality of the anesthetic area was assessed. The anesthetic level was assessed. Measurement of RR interval,
QT interval, QTc interval, QTD, QTcD, Tp-Te, Tp-Te/ $\mathrm{QT}$, and $\mathrm{Tp}-\mathrm{Te} / \mathrm{QTc}$ was performed before the epidural injection (baseline), as well as at 0 minutes (T0), 1 minute (T1), 3 minutes (T3), 5 minutes (T5), 10 minutes (T10), 15 minutes (T15), 20 minutes (T20), 25 minutes (T25), and 30 minutes (T30) after the epidural injection. From the ECG, consecutive beat-to-beat data were digitally recorded at a sampling rate of $2 \mathrm{msec}$. QT intervals were measured by using QTD-1 ${ }^{\text {TM }}$ (Fukuda Denshi Co. Ltd), which detected the onset of the $\mathrm{Q}$ wave and the end of the $\mathrm{T}$ wave. This technique determines the onset of the $\mathrm{Q}$ wave as the intersection of a threshold level with the differential of the $Q$ wave and the end of the $T$ wave as the intersection of a threshold level with the differential of the $\mathrm{T}$ wave. The software used for the differential threshold measurement technique has been previously described in detail. ${ }^{6,7}$ QT intervals were measured in all 12-lead ECGs and corrected by using Bazett's formula $(\mathrm{QTc}=\mathrm{QT} / 2 \sqrt{\mathrm{RR}})$. QTD was defined as the difference between the maximum and minimum average QT interval in 12-lead ECG. Similarly, QTcD was defined as the difference between the maximum and minimum average QTc interval. Tp-Te was determined from the peak of the $T$ wave to the end of the $\mathrm{T}$ wave in lead II. The average value of data derived from three successive beats for each lead was used for analysis. Leads in which the end of the $T$ wave could not be clearly detected were excluded from this study. The RR interval, sBP, dBP, QT, QTc, QTD, QTcD, Tp-Te, Tp-Te/ $\mathrm{QT}$, and Tp-Te/QTc were analyzed.

\section{Statistical analyses}

Based on a previous study, ${ }^{8}$ a sample size of 17 subjects was considered adequate to detect a difference of $20 \mathrm{msec}$ in QTD, compared with baseline, at a power of $80 \%, \alpha=0.05$. Data are expressed as mean $\pm \mathrm{SD}$. Changes in sBP, dBP, RR interval, QT, QTc, QTD, QTcD, Tp-Te, Tp-Te/QT, and Tp$\mathrm{Te} / \mathrm{QTc}$ were analyzed by using one-way ANOVA. When a significant overall effect was detected, Dunnett's post hoc test was conducted. In all analyses, the probability to detect a difference was set at the $5 \%$ level $(P<0.05)$.

\section{Results}

Table 1 shows the patient characteristics. Twenty-three patients were enrolled in this study. Nine patients received medication for hypertension (eplerenone or amlodipine) and one patient received medication for diabetes mellitus (voglibose and metformin). No complications were observed in the present study. No abnormalities were found in the levels of preoperative serum sodium, potassium, and calcium. 
Table 2 shows the variables in all measurement values throughout the observation. No patients exhibited abnormalities of RR, sBP, dBP, QT, QTc, QTD, QTcD, Tp-Te, $\mathrm{Tp}-\mathrm{Te} / \mathrm{QT}$, or Tp-Te/QTc at baseline (RR: $876 \pm 99 \mathrm{msec}$, QT: $380 \pm 28 \mathrm{msec}$, QTc: $408 \pm 26 \mathrm{msec}$, QTD: $31 \pm 15 \mathrm{msec}$, QTcD: $33 \pm 15 \mathrm{msec}, \mathrm{Tp}-\mathrm{Te}: 71 \pm 9$, Tp-Te/QT: $0.187 \pm 0.017$, Tp-Te/QTc: $0.176 \pm 0.021)$. There was no significant change in RR interval after epidural injection of $1 \%$ mepivacaine. In contrast, sBP significantly decreased at $\mathrm{T} 10$ and $\mathrm{T} 15$, compared with baseline, after epidural injection (baseline: 125 $\pm 14 \mathrm{mmHg}$, T10: 113 $\pm 13 \mathrm{mmHg}$, and T15: $111 \pm 14$ $\mathrm{mmHg}$, respectively; $P<0.05$ ). In contrast, there was no significant difference in dBP. There were no changes in QT, QTc, QTD, QTcD, Tp-Te, Tp-Te/QT, or Tp-Te/QTc during the study.

\section{Discussion}

A previous study demonstrated the reduction of QTD and $\mathrm{Tp}-\mathrm{Te}$ by thoracic epidural anesthesia with $0.5 \%$ bupivacaine due to the blockade of sympathetic fibers innervating the heart. ${ }^{5}$ The present study revealed the safety of thoracic epidural sympathetic block by $1 \%$ mepivacaine on cardiac repolarization, compared with right $\mathrm{SGB}$, which was previously described.

QTD has been known as a marker of ventricular arrhythmias. A previous study demonstrated that QTcD was increased in patients with arrhythmogenic QT prolongation, such as Romano Ward syndrome or Jervell and Lange-Nielsen syndrome, compared to patients with QT prolongation by sotalol. ${ }^{9}$ Moreover, QT dispersion and QTc dispersion were significantly prolonged in acute myocardial infarction patients with ventricular fibrillation compared to patients with unstable angina. ${ }^{10}$ These results suggest the efficacy of QTcD to predict the fatal ventricular arrhythmia or sudden cardiac death.

Table I Patient characteristics

\begin{tabular}{|l|l|}
\hline Characteristics & $\mathbf{n}$ \\
\hline Sex (male/female) & $17 / 6$ \\
Age (years) & $61.1 \pm 13$ \\
Height $(\mathrm{cm})$ & $161.4 \pm 8.1$ \\
Weight $(\mathrm{kg})$ & $60.1 \pm 11.9$ \\
$\mathrm{BMI}\left(\mathrm{kg} / \mathrm{m}^{2}\right)$ & $23.0 \pm 3.7$ \\
Sodium $(\mathrm{mEq} / \mathrm{L})$ & $139.7 \pm 3.1$ \\
Potassium $(\mathrm{mEq} / \mathrm{L})$ & $4.3 \pm 0.3$ \\
Calcium $(\mathrm{mg} / \mathrm{dL})$ & $9.1 \pm 0.3$ \\
Medications & \\
Hypertension & 9 \\
Diabetes mellitus & $\mathrm{I}$ \\
\hline
\end{tabular}

Note: Data are presented as mean \pm SD. Abbreviation: BMI, body mass index.

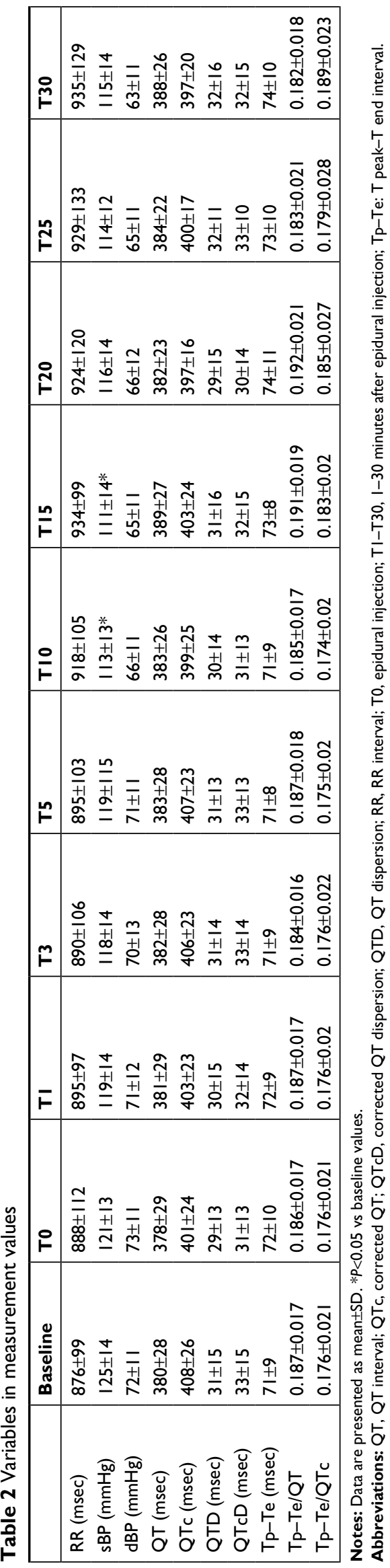


Thoracic epidural sympathetic block is an option for outpatients to improve intractable pain or upper limb blood flow. Similarly, SGB is commonly used for pain relief or disturbances in blood flow. A previous report suggested that right SGB significantly increased QTD, compared with left SGB. ${ }^{1}$ This difference may be caused by the laterality of the distribution of sympathetic nerves. Notably, sympathetic fibers from the right stellate ganglion mainly distribute to the anterior walls of the ventricles, whereas those from the left stellate ganglion distribute to the posterior walls. ${ }^{11}$ Rogers et al reported that sympathetic accelerator fibers are more prominent on the right ganglion than on the left ganglion. ${ }^{12}$ It has been demonstrated that autonomic innervation to the sinus node is predominantly from the right-side stellate ganglion; ${ }^{13}$ furthermore, right-side SGB attenuated both sympathetic and parasympathetic activities. These findings imply that the right stellate ganglion may assume a key role in homogenization of ventricular repolarization and maintenance of cardiac electric stability, compared with the left stellate ganglion. Therefore, right-side SGB may prolong QT dispersion, which is an index of heterogeneity in ventricular repolarization. Thus, right SGB should be carefully selected as a treatment, especially in patients with cardiovascular disease.

Previous studies suggested that thoracic epidural anesthesia has no effect on cardiac function. ${ }^{14,15}$ Regarding QT interval, Owczuk et al reported that thoracic epidural anesthesia induced by a long-acting agent, $0.5 \%$ bupivacaine (a maximum concentration for epidural anesthesia), significantly shortened the QT interval and Tp-Te by blocking preganglionic sympathetic fibers (Th1-Th4) that innervate the heart. ${ }^{5}$ In patients with ischemic cardiomyopathy, thoracic epidural block by $0.5 \%$ bupivacaine could block cardiac sympathetic overactivity and normalize myocardial blood flow. ${ }^{16}$ In our present results, thoracic epidural block by short-acting anesthetics with $1 \%$ mepivacaine (a low dose for epidural anesthesia) slightly prolonged RR interval and reduced sBP, but did not affect QT or QTD. Thus, epidural $1 \%$ mepivacaine did not predominantly block sympathetic cardiac fibers, compared with $0.5 \%$ bupivacaine. Therefore, thoracic epidural block by local anesthetics, which blocks sympathetic nerve systems, did not alter hemodynamic status and cardiac repolarization, as determined by QTD.

$\mathrm{Tp}-\mathrm{Te}$ reflects ventricular repolarization and is regarded as a noninvasive arrhythmogenic index. This marker is associated with a high risk of sudden cardiac death. ${ }^{12,17}$ In patients who received appropriate defibrillator therapy, $\mathrm{Tp}-\mathrm{Te}$ and $\mathrm{Tp}-\mathrm{Te} /$ QT ratio were significantly increased. Moreover, in patients with nonsustained ventricular tachycardia, $\mathrm{Tp}-\mathrm{Te} / \mathrm{QT}$ ratio was significantly increased. ${ }^{17} \mathrm{The} \mathrm{Tp}-\mathrm{Te} / \mathrm{QT}$ ratio eliminates the confounding effects of variabilities in heart rate and QT interval. ${ }^{2}$ Therefore, $\mathrm{Tp}-\mathrm{Te} / \mathrm{QT}$ ratio is considered a more sensitive index of ventricular arrhythmia, compared with $\mathrm{Tp}-\mathrm{Te}$ interval. A Tp-Te/QT ratio $>0.25$ is strongly associated with the risk of ventricular arrhythmias in implantable cardioverter defibrillators therapy ${ }^{17} \mathrm{In}$ our study, Tp-Te, Tp-Te/QT, and Tp$\mathrm{Te} / \mathrm{QTc}$ did not significantly change during the measurement. The epidural block by $1 \%$ mepivacaine did not alter TDR.

No previous study assessed the effect of epidural sympathetic block by $1 \%$ mepivacaine on QT dispersion. Hence, to determine the sample size, we applied our previous study, ${ }^{8}$ which evaluated the effect of reversal of neuromuscular blockade, but not local anesthesia, on QT dispersion.

Our study has several limitations. The present observations were conducted under conscious conditions; the sympathetic nerve systems described by QTD or Tp-Te could be easily changed by psychological factors under such conditions.

In our study, cardiac function was not evaluated using echocardiography. Our primary endpoint was the change of cardiac repolarization, which is a marker of ventricular arrhythmia. However, a detailed assessment of hemodynamic changes with echocardiography is essential to assure the safety of epidural block in each patient.

In conclusion, our results show that thoracic epidural sympathetic block by $1 \%$ mepivacaine did not affect $\mathrm{Tp}-\mathrm{Te}$, $\mathrm{Tp}-\mathrm{Te} / \mathrm{QT}$, or $\mathrm{Tp}-\mathrm{Te} / \mathrm{QTc}$, which is a precursor for fatal ventricular arrhythmias. Therefore, thoracic sympathetic block by $1 \%$ mepivacaine is a safer option, compared with right SGB, in terms of cardiac repolarization.

\section{Data sharing statement}

The individual de-identified data generated or analyzed during the current study are available from the corresponding author on reasonable request beginning 9-36 months following publication.

\section{Disclosure}

The authors report no conflicts of interest in this work.

\section{References}

1. Egawa H, Okuda Y, Kitajima T, Minami J. Assessment of QT interval and QT dispersion following stellate ganglion block using computerized measurements. Reg Anesth Pain Med. 2001;26(6):539-544.

2. Gupta P, Patel C, Patel H, et al. T(p-e)/QT ratio as an index of arrhythmogenesis. J Electrocardiol. 2008;41(6):567-574.

3. Kors JA, Ritsema van Eck HJ, van Herpen G. The meaning of the Tp-Te interval and its diagnostic value. J Electrocardiol. 2008;41(6):575-580. 
4. Kilicaslan F, Tokatli A, Ozdag F, et al. Tp-e interval, Tp-e/QT ratio, and $\mathrm{Tp}-\mathrm{e} / \mathrm{QTc}$ ratio are prolonged in patients with moderate and severe obstructive sleep apnea. Pacing Clin Electrophysiol. 2012;35(8):966-972.

5. Owczuk R, Steffek M, Wujtewicz MA, et al. Effects of thoracic epidural anaesthesia on cardiac repolarization. Clin Exp Pharmacol Physiol. 2009;36(9):880-883.

6. Mclaughlin NB, Campbell RW, Murray A. Accuracy of four automatic QT measurement techniques in cardiac patients and healthy subjects. Heart. 1996;76(5):422-426.

7. Xue Q, Reddy S. Algorithms for computerized QT analysis. J Electrocardiol. 1998;30 Suppl:181-186.

8. Nagashima S, Takasusuki T, Yamaguchi S, Hamaguchi S. Effects of neostigmine and sugammadex on QT interval and QT dispersion. Dokkyo J Med Sci. 2016;43:15-22.

9. Day CP, Mccomb JM, Campbell RW. QT dispersion: an indication of arrhythmia risk in patients with long QT intervals. Br Heart J. 1990;63(6):342-344.

10. Higham PD, Furniss SS, Campbell RW. QT dispersion and components of the QT interval in ischaemia and infarction. Br Heart J. 1995;73(1):32-36.

11. Wurster RD. Spinal Sympathetic Control of the Heart. Neural Regulation of the Heart. Oxford: Oxford University Press. 1977;213.
12. Rogers MC, Battit G, Mcpeek B, Todd D. Lateralization of sympathetic control of the human sinus node: ECG changes of stellate ganglion block. Anesthesiology. 1978;48(2):139-141.

13. Fujiki A, Masuda A, Inoue H. Effects of unilateral stellate ganglion block on the spectral characteristics of heart rate variability. Jpn Circ J. 1999;63(11):854-858.

14. Gurses E, Berk D, Sungurtekin H, Mete A, Serin S. Effects of high thoracic epidural anesthesia on mixed venous oxygen saturation in coronary artery bypass grafting surgery. Med Sci Monit. 2013;19:222-229.

15. Mohamad MF, Mohammad MA, Hetta DF, Ahmed EH, Obiedallah AA, Elzohry AAM. Thoracic epidural analgesia reduces myocardial injury in ischemic patients undergoing major abdominal cancer surgery. J Pain Res. 2017;10:887-895.

16. Nygård E, Kofoed KF, Freiberg J, et al. Effects of high thoracic epidural analgesia on myocardial blood flow in patients with ischemic heart disease. Circulation. 2005;111(17):2165-2170.

17. Barbhaiya C, Po JR, Hanon S, Schweitzer P. Tpeak-Tend and Tpeak-Tend/QT ratio as markers of ventricular arrhythmia risk in cardiac resynchronization therapy patients. Pacing Clin Electrophysiol. 2013;36(1):103-108.
Local and Regional Anesthesia

\section{Publish your work in this journal}

Local and Regional Anesthesia is an international, peer-reviewed open access journal publishing on the development, pharmacology, delivery and targeting and clinical use of local and regional anesthetics and analgesics. The journal is included in PubMed, and welcomes submitted papers covering original research, basic science, clinical studies,

\section{Dovepress}

reviews and evaluations, guidelines, expert opinion and commentary, case reports and extended reports. The manuscript management system is completely online and includes a very quick and fair peer-review system, which is all easy to use. Visit http://www.dovepress.com/ testimonials.php to read real quotes from published authors. 